\title{
Dog Breed Prediction using Convolutional Neural Network
}

\author{
Sneha I. Kadari, Shubhada S. Kulkarni, Sharada G. Kulkarni
}

\begin{abstract}
Deep learning gives the strength on the way to train algorithms model that can handle the difficulties of info classification also prediction grounded on totally on arising information as of raw information. Convolutional Neural Networks (CNNs) gives single often used method for image classification and detection. In this exertion, we define a CNNbased approach for spotting dogs in per chance complex images and due to this fact reflect inconsideration on the identification of the one of kinds of dog breed. The experimental outcome analysis supported the standard metrics and thus the graphical representation confirms that the algorithm (CNN) gives good analysis accuracy for all the tested datasets.

Keywords: Machine Learning, Tensorflow, Classification, CNN, Deep Learning
\end{abstract}

\section{INTRODUCTION}

In machine learning, convolutional Neural Network (CNN) is complicated feed forward neural networks. CNNs are used for image classification and recognition due to its excessive accuracy. The CNN follows a hierarchical model which struggles on constructing a network, form of a funnel, and eventually offers out a definitely related layer the place all the neurons are linked to each and every different and consequently the output is processed A computer learns to classify images, text and sound. The pc is trained with large image datasets then it changes the pixel value of the image to an indoor representation, where the classifier can detect patterns on the input image. During this project, a deep learning convolutional neural network supported Tensorflow is deployed using Nodejs for binary image classification. Convolutional neural networks became universal in computer vision ever meanwhile popularized deep convolutional neural networks by way of prevailing the ImageNet Task the overall tendency has remained to form deeper and greater intricate networks to recognize greater accuracy. In our project, a deep learning convolutional network supported Tensorflow is deployed using node for binary image classification. We present a Tensorflow model called MobileNet for mobile and embedded vision applications. MobileNet are modernized building that needs depth wise divisible convolutions to structure thin deep neural networks. We present experiments on source and accuracy tradeoffs and exhibit lively achievements in contrast to different appreciated models on ImageNet classification

Revised Manuscript Received on May 10, 2020.

Sneha I. Kadari*, Computer Science and Engineering, KLS Gogte Institute of Technology, Belagavi, India. (E-mail: kadarisneha@gmail.com)

Shubhada S. Kulkarni, Professor Computer Science and Engineering, KLS Gogte Institute of Technology, India. (E-mail: sskulkarni@git.edu) KLS Gogte Institute of Technology, Belagavi, India. (E-mail: sharadakulkarni@git.edu)

(C) The Authors. Published by Blue Eyes Intelligence Engineering and Sciences Publication (BEIESP). This is an open access article under the CC BY-NC-ND license (http://creativecommons.org/licenses/by-nc-nd/4.0/)
Sharada G. Kulkarni, Professor Computer Science and Engineering,

\section{OBJECTIVES}

In this paper our main objective is to implement an Image Classification with Deep Learning and Convolutional Neural Networks using Tensorflow.

- Computers nowadays cannot only acknowledge pictures; however, they will additionally describe the assorted parts in photos and create transient sentences. This is done by $\mathrm{CNN}$ that learns patterns that occur in pictures.

O To train the Convolutional Neural Networks using one of the largest databases of labelled images. Deep learning frameworks such as TensorFlow etc.

\section{LITERATURE SURVEY}

Deep learning has been around as a part of the machine learning world since a long time. The actual lineage of deep learning according to the authors Foote, 2017, can be tracked right back to 1943 where an attempt was made by Walter Pitts and Warren McCulloch to design a computer typical which was founded on the neural network of the human intellect. However, "the earliest efforts in developing Deep Learning algorithms came from Alexey Grigoryevich Ivakhnenko (developed the Group Method of Data Handling) and Valentin Grigor'evich Lapa (author of Cybernetics and Forecasting Techniques) in 1965" Foote, 2017 [1]. Deep Learning is a part of machine learning which uses multi-layer neural networks for performing operations. Each layer inside the neural network consists of several neurons which are all connected in such a way that they can communicate with each other. This neuron was devised with the hope that it would work in a comparable way such as the neuron in a human intelligence would work. Here the neuron would try and calculate the weighted average of the values i.e. the input signal and an output signal that is transmitted by the connected neuron Arora et, el , 2015 [2]. Nokwon Jeong, Soosun Cho, 2017 [3], the authors try image classification on the Instagram images where their focus hinges on evaluating the competitive power of deep learning for classification of real time social networking images. The authors Nokwon Jeong, Soosun Cho, 2017 in their study also look at the performance of pre-existing CNN frameworks such as AlexNet and ResNet and how well they perform on the ImageNet dataset which showed outstanding capabilities.

\section{SYSTEM DESIGN}

The sketch of planned system is shown in fig. 4.1. Apiece block of recommended sketch is clearly labelled and represents processing steps. 


\section{Dog Breed Prediction using Convolutional Neural Network}

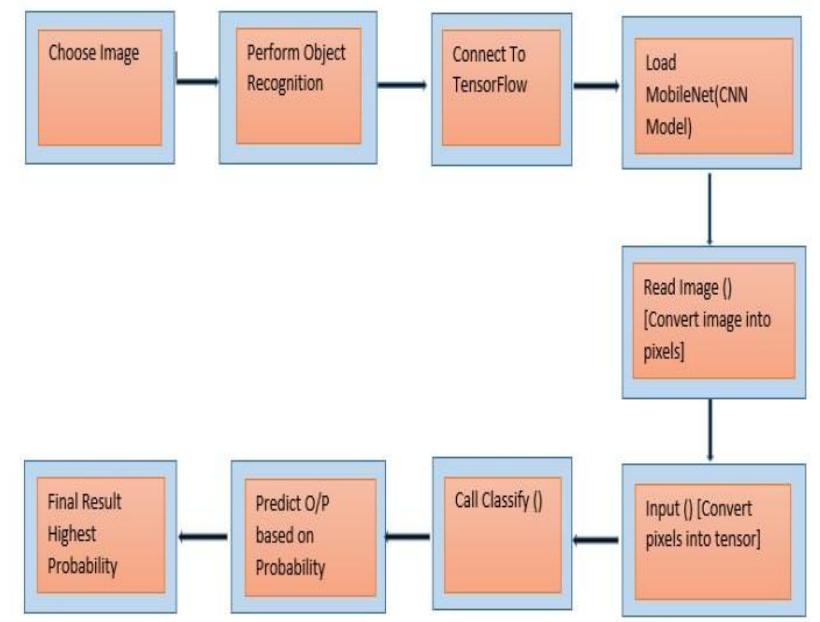

Fig 4.1: System Design

In first step, we choose an image as an input in which we want to predict the object. In the second step, we perform object recognition which will connect to the Tensorflow which contains 1000 images of different objects used for training as mentioned in third step. In the fourth step the MobileNet Model is loaded which is CNN (convolution Neural Network) model. CNN with two convolutional layers, pick out special combination of activation features and classifiers for comparison purpose. Test the system with training datasets respectively. Then in fifth step the input image is given as input parameter to ReadImage()which converts the image into pixels. In the sixth stepInput() is executed which converts the pixel image into tensor and the in final step this tensor image is given as input parameter to classify function which predict the output based on the probability from which we consider the highest probability value as a best predicted object.

\section{METHODOLOGY}

\section{A. Neural Network:}

A computational models that everything in a related mode to the neurons in the human intellect. Apiece neuron takings and input, achieves nearly actions then passes the output to the subsequent neuron.

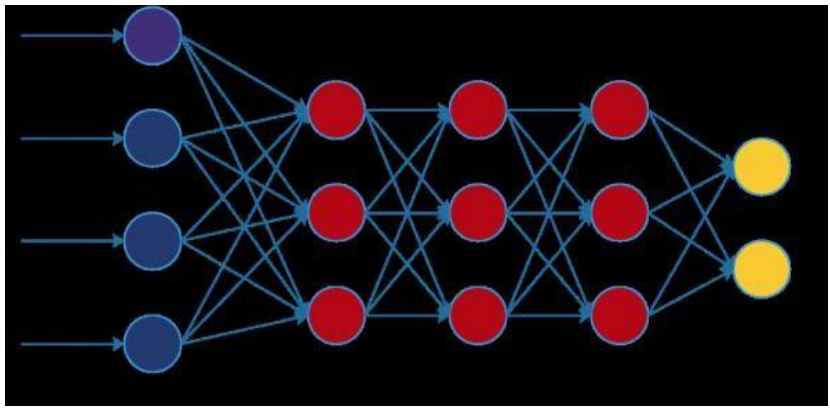

Fig 5.1: Neural Network representation

For example, we are working to provide an explanation for the device to identify images and order them into single of these 10 types.

airplane
automobile
bird
cat
deer
dog
frog
horse
ship
truck

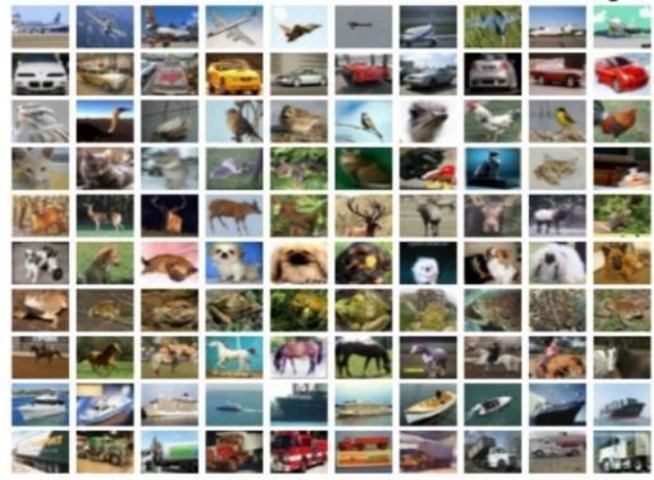

Fig 5.2: Sample Images Dataset

To ensure, we initial want to prepare the device how a bird, a cat, a horse, etc. seem to be similar earlier than it being capable to identify an original object. The additional birds the computer gets, the greater it receives in finding birds. This is identified as supervised learning. We can raise this challenge in means of tagging the pictures, the computer resolve begins distinguishing forms existing in bird pic that are remain away from different ones and can begin constructing its private recognition. We are at work to usage TensorFlow on the way to write the program. TensorFlow is an open source deep learning structure build by Google that offers builders coarse deal with concluded a piece neuron recognized as a "node" in TensorFlow. Thus, you can modify the weightiness and manipulates optimum presentation. TensorFlow has many built-in libraries also has a super community; we will be capable to open source executions for effectively any deep learning topic.

\section{B. Convolutional Neural Network:}

Convolutional Neural Network is an exceptional kind of bolster advancing artificial neural network, which stimulated through visual cortex. In CNN, the neuron in a layer is solely related to a small area of the layer earlier than it, alternatively of all the neurons in a entirely related manner, so CNN manage fewer quantities of weights and much less number of neurons.

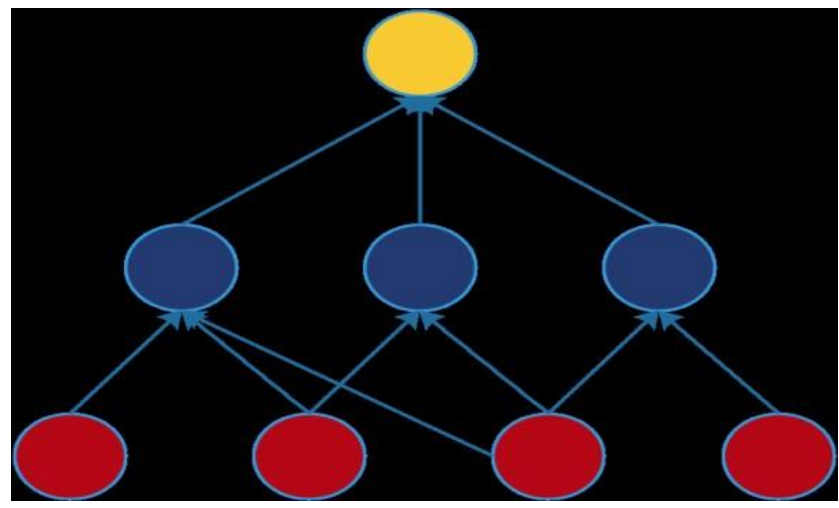

Fig 5.3: Convolutional Neural Network The main support of this web-based application is:

\subsubsection{AngularJS:}

AngularJS is an open-source and completely free and licensed under apache and maintained by Google then in a public of persons and company to contract with some of the valuations come across in

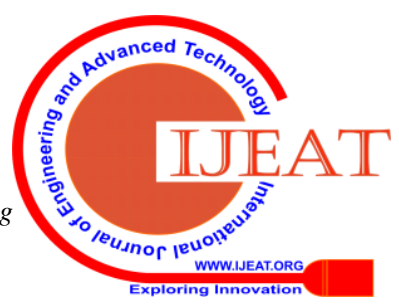


improving single-page applications.

Where the main intended to make clear each the progress and the challenging of such applications via given a basis for client-side model-view-controller (MVC) and modelview view model (MVVM) architecture, sideways with components normally used web applications.

The AngularJS framework entirety via look the Hypertext mark-up language page, which has brought custom HTML attributes, surrounded into it. Angular describe these attributes as directions to bind input or output component of the web page to a model that's label through well-known JavaScript variables. The values of these JavaScript variables are frequently established inside the code, or retrieved from static or dynamic JSON resources.

\subsubsection{Tensor flow:}

It is an open-source library and used as the back-end library in developing our model since it's widely used for machine learning applications such as neural networks you can use to define, train, and run machine learning models exactly within the browser, using JavaScript and a high-level layers API. It is designed for handling data that's kept within the tensor format.

\subsubsection{Node.js:}

Node.js is a JavaScript runtime environment includes everything you need to execute a program written in JavaScript.
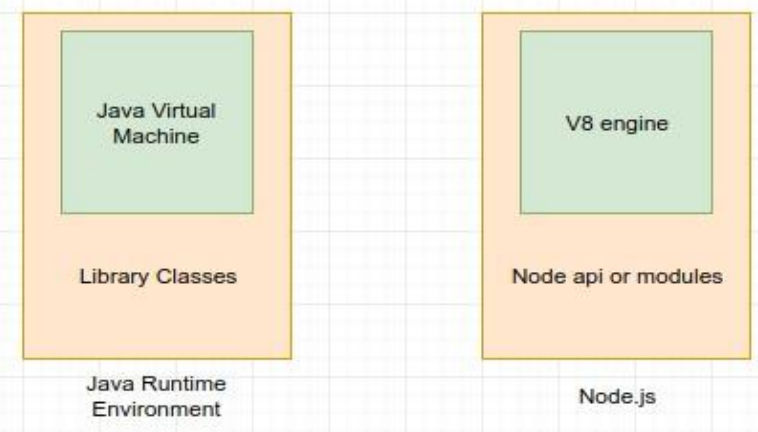

Fig 5.1.3: Node run environment

Node.js got here into presence when the first builder of JavaScript persevered it from something that you'll run inside browser on your device as a standalone. But Now you'll do away greater with JavaScript used to create interactive effects within web browsers

Now JavaScript has the plausible to strive to do matters that different scripting languages like Python can do. Single then the dissimilar your browser JavaScript and Node.js run on the V8 JavaScript runtime machine. In order to v8 translates JavaScript code into quicker. Machine code is low-level code which the computer can run except having to first interpret it. Node.js eliminates the waiting, and easily continues with subsequent request.

\subsubsection{NPM (Node package manager):}

These libraries are constructing by means of super community they may clear up maximum wide-spread issues. NPM (Node package manager) has packages you could use for your apps to form your improvement quicker and adequate.

\section{CONDITION:}

\section{Necessity three things:}

- It bundles components that derived on or after Node.js like file system and Hypertext transfer protocol.

- It bundles libraries like Express and Mongoose that absolutely installation from Node package manager.

- It lets you in to take your own files and combined the project and it could also be a function, and it agrees to take a restriction "path" and return exposed as module.

\subsubsection{EXPRESS.js:}

Express.js ought to additionally be an web utility shape forNode.js, freed as complimentary and open-source software program beneath the MIT License. It is planned for create net functions also APIs. It has been referred to as the the de facto standard server shape for Node.js.

\subsubsection{BOOTSTRAP:}

Bootstrap is that the tropical HTML, CSS, and JS context meant for designing open, portable primary projects arranged the online. As well, Bootstrap involves jQuery to function. jQuery is a particularly interesting and broadly used JavaScript library, that together make simpler and enhances cross browser suitable toward JavaScript.

Some of the customs that agendas can aid you:

- Avoid recurrence amongst projects

- Exploit receptive strategy to agree your website to familiarize to many screen scopes like - portable, desktop, and the lot in amongst

- Enhance reliability to project and code among schemes and among manufacturers

- $\quad$ Fast and effortlessly model innovative designs

- Guarantee cross-browser suitable

\subsubsection{MongoDB:}

- MongoDB is an open supply database that makes use of document-oriented data model and a nonstructured command language. It's one amongst the effective NoSQL structure and databases around nowadays.

- Being a NoSQL capability it does not no longer use the rows and columns that we so much associate with the relational database management. It is an architecture that's constructed on collections and documents. The fundamental unit of knowledge for the duration of this database subsist of a group of key value pairs.

- It permits to possess different fields and structures from documents. This database makes use of document storage format called BSON which can be a binary kind of JSON style documents.

- MongoDB follows may be a highly flexible that allows you to lets you blend and store information of multivariate kinds except having to compromise on the effective indexing options, data access and validation rules. 


\section{Dog Breed Prediction using Convolutional Neural Network}

There's is no downtime as soon as you when you prefer to dynamically adapt the schemas. So, what it means you'll pay extra attention on making your data work harder rather than instead of spending longer on getting ready the information for the database.

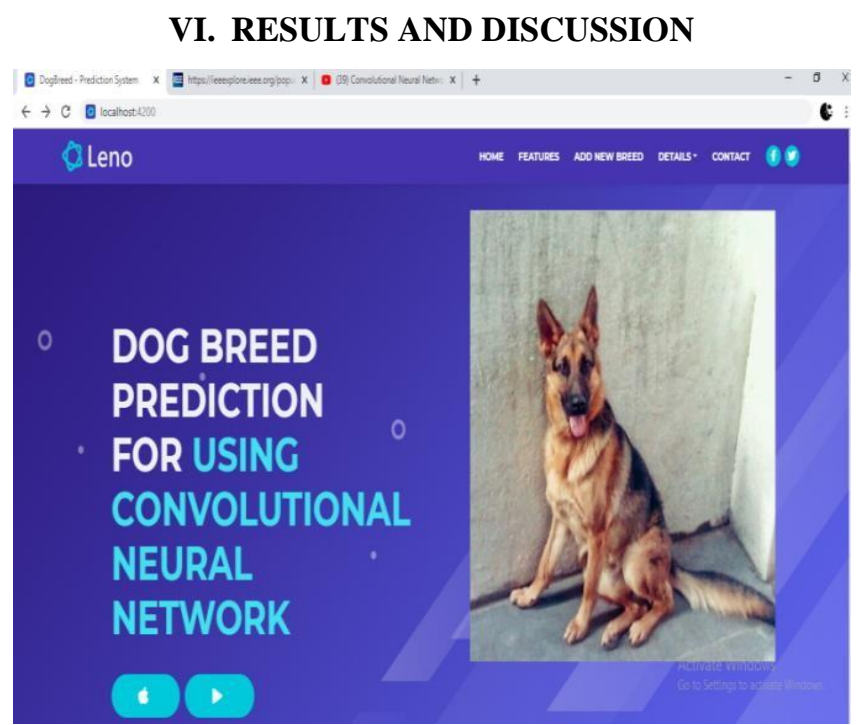

Fig 6.1: Home page

The above figure illustrates the user interface plan.

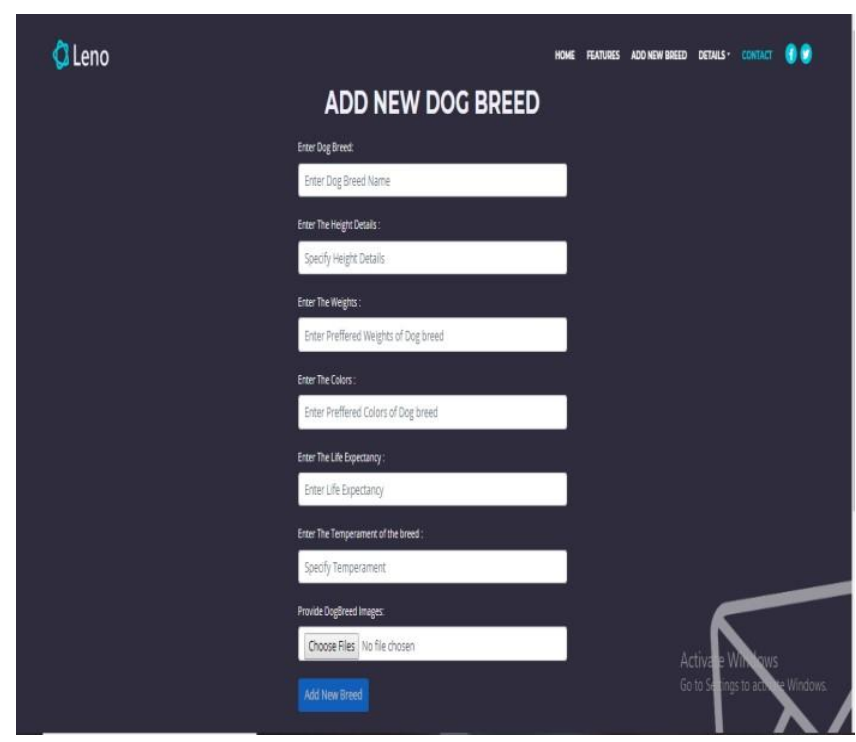

Fig 6.2: User input form

The above figure depicts the user interface design with the user input form. Through this form, we select the image file as an input to predict the object present in it.

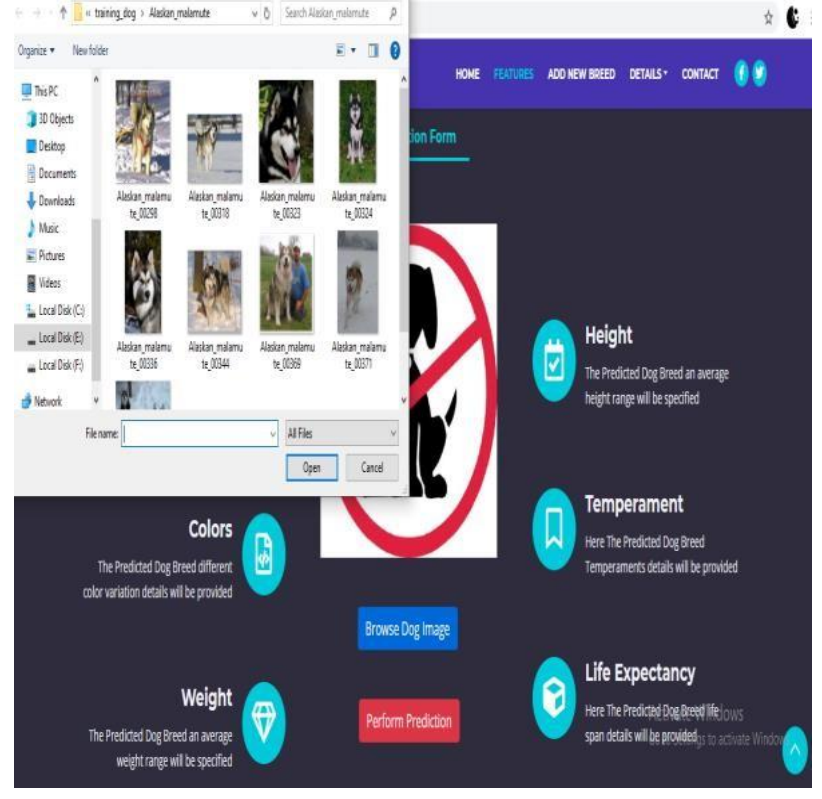

Fig 6.3: user input form with dialogue box

The above figure depicts that we can choose an image of our choice via open dialogue box from any path location.

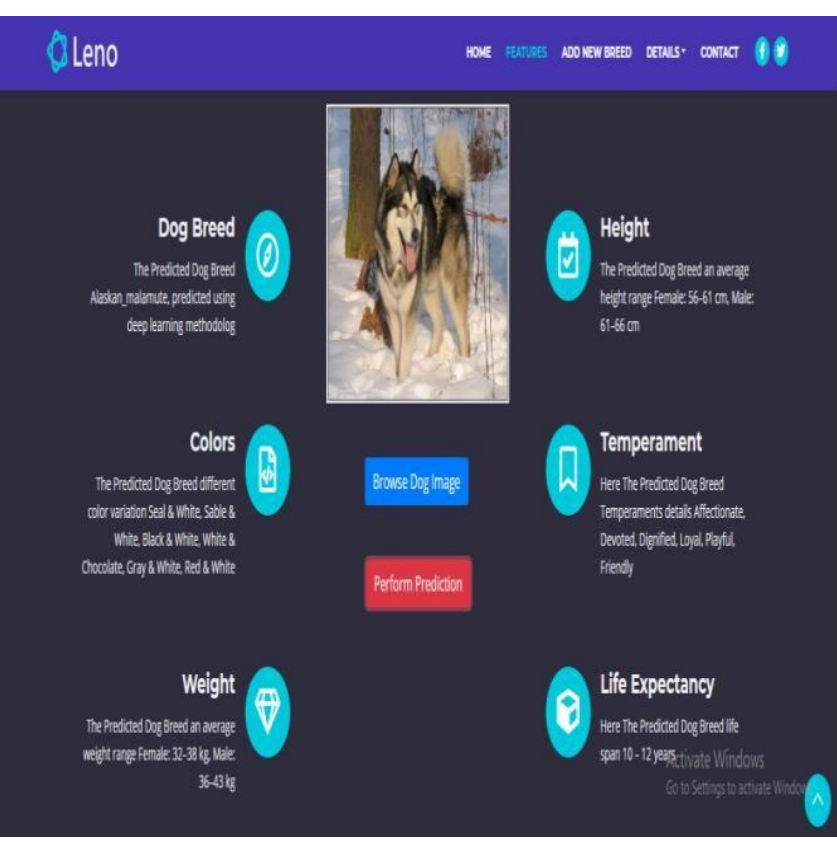

Fig 6.4: prediction of result

The above figure shows that the prediction is done on the image selected. The present object detected in the image is Alaskan malamute dog. And dog details.

\section{DISCUSSION}

This segment grants the conclusion of the prediction accuracies gained using CNN procedure arranged various standard datasets. The outcomes remain existing by means of the estimate accurateness in proportion for inside train information and test data separately. Along with the prediction accuracy percentage values, MSE (Mean Squared Error) graph is also given. The Graphs exhibit the exchange of MSE by regard on the way to the training epochs. MSE metric is the easiest and commonly used quality metric.
Blue Eyes Intelligence Engineering $\&$ Sciences Publication

(C) Copyright: All rights reserved.

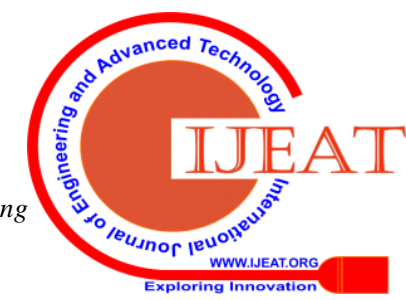


It is the suggest of the squared difference between original and trained approximation MSE shows the shut nearness among basic pictures then the closing recreated output. The thought right now remains toward usage sufficient quantity of epochs that would conclusion in low MSE, high arrangement accurateness then by minimum for training the network. The network remains examined on number of different datasets, in flip every dataset is examined for various number of iterations (epochs).

Figure 6.5, 6.6 and 6.7 show variation of MSE for 200, 300 and 500 epochs respectively. From the graphs below, we infer that the MSE cut down considerably as the epoch value changes from 200 to 300. However, the MSE value undergoes minimum variation, as we change the number of epochs from 300 to 500 .

A
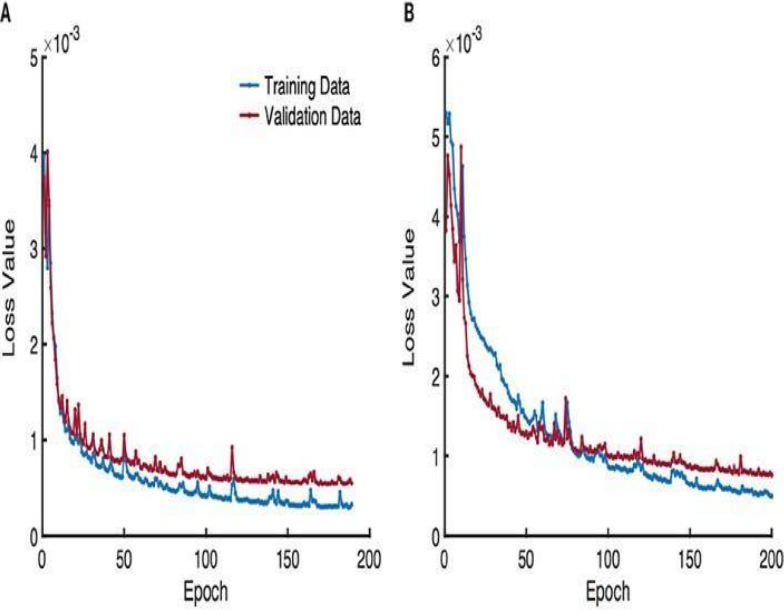

Fig 6.5: Variation of MSE (200 epochs)

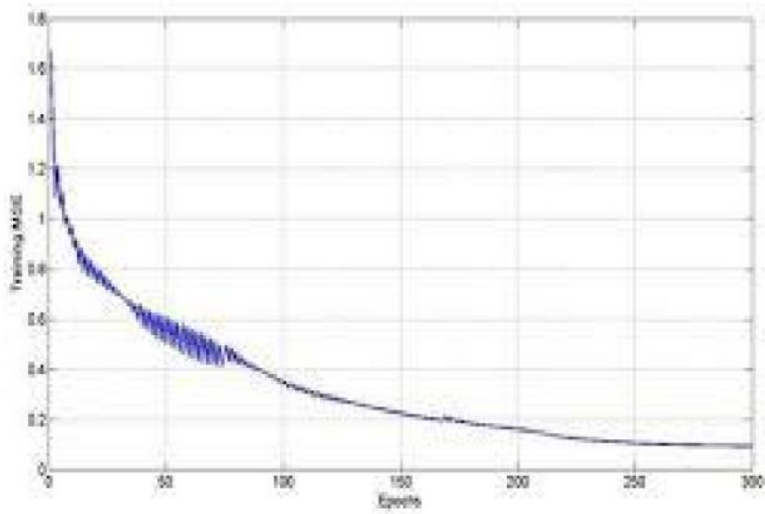

Fig 6.6: Variation of MSE (300 epochs)

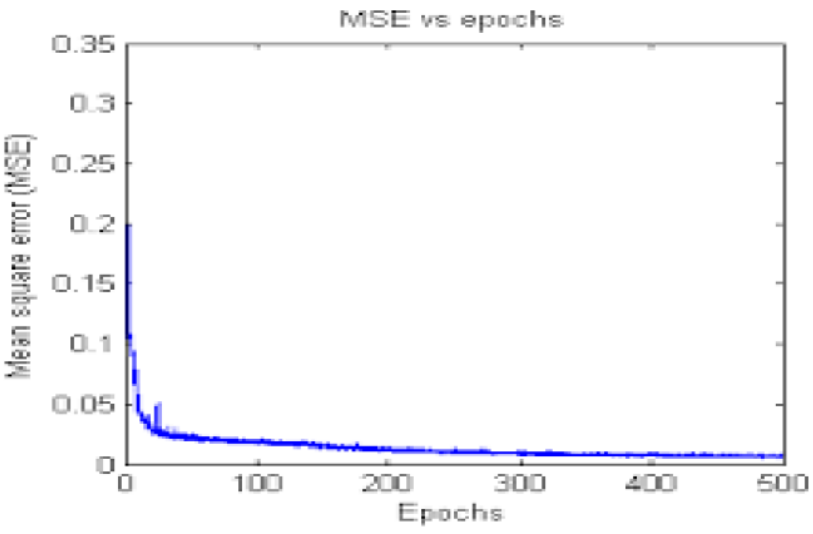

Fig 6.7: Variation of MSE (500 epochs)

\section{VII.CONCLUSION}

Convolutional neural network is a learning method for data analysis and predictions, now days it also become very popular for image classification problems. Dog breed prediction of deep learning developed using convolutional neural network is to predict the breed of hundred images in taking their images as input. Usage transfer learning on the way to build model that make output and around to hundreds of dissimilar dog types. The results were pretty good for the images the model was shown. The algorithm was able to identify dog breeds quite exactly. Transfer learning takes a excessive choice in the upcoming in joining a prebuilt model by the model we created.

\section{REFERENCES}

1. Keith D. Foote, (2017). A Brief History of Deep Learning DATAVERSITY. [online] DATAVERSITY.Available:https://www.dataversity. net/briefhistory-deep-learning/

2. Arora, A., Candel, A., Lanford, J., LeDell, E. and Parmar, V. (2015) Deep Learning with H2O. 3rd ed. [ebook]Availableat:https://h2orelease.s3.amazonaws. com/h2o/master/3190/docswebsite/h2odocs/booklets/ DeepLearning_Vignette.pdf

3. Nokwon Jeong, Soosun Cho (2017) 'Instagram image classification with Deep Learning'.

4. J. Salamon and J. P. Bello, Deep Convolutional Neural Networks and Data Augmentation for Environmental Sound Classification, no. November, pp. 15, 2016.

5. H.C. Shin, L. Lu, L. Kim, A. Seff, J. Yao, and R. M. Sum- mers Interleaved text/image deep mining on a very large- scale radiology database. In Proceedings of the IEEE Con-ference on Computer Vision and Pattern Recognition , pages 10901099, 2015

6. A. Krizhevsky, I. Sutskever, and G. E. Hinton, ImageNet Classification with Deep Convolutional Neural Networks, Adv. Neural Inf. Process. Syst., pp. 19, 2012

7. K. Chatfield, K. Simonyan, A. Vedaldi, and A. Zisserman, Return of the Devil in the Details: Delving Deep into Convolutional Nets, arXiv Prepr. ArXiv , pp. 111, 2014 